\title{
sciendo
}

\section{Work-rate Analysis of Substitute Players in Professional Soccer: Analysis of Seasonal Variations}

\author{
by \\ Alexis Padrón-Cabo ${ }^{1}$,Ezequiel Rey ${ }^{1}$, Benjamín Vidal ${ }^{1}$ Javier García-Nuñez ${ }^{2}$
}

The aims of this study were to evaluate physical performance of substitute players versus those replaced or completing the entire match, determine physical performance of substitute players across different playing positions and examine variations in match-related running performance in substitute players throughout the entire competitive season. The sample was composed of 943 observations of professional players who participated in the first division of the Spanish League (La Liga) during the 2014-2015 season. The players were divided into three different groups: players who completed the entire match $(n=519)$, players who were replaced $(n=212)$ and substitute players $(n=212)$. Substitute players covered greater distances at medium and high intensity compared to the players who played the entire match and those who were replaced. Position-specific trends indicated that attackers and central midfielder increased the distance covered at high-intensity running compared to their peers who played the whole match. During the competitive season, it was observed that substitute players attained greater match running performance during the mid-season period, allowing them to cover more distance for different variables of running performance compared to the start and end of the season.

Key words: substitute's performance analysis, seasonal variations, playing position, high-intensity running.

\section{Introduction}

While in many team sports coaches are allowed to make an unlimited amount of substitutions, in soccer, a maximum of 3 substitutions are granted during an official match, in accordance with Rule 3 of the Fédération Internationale de Football Association regulation (FIFA, 2014). A substitution is when a player is brought on to the pitch during a match in exchange for an existing player, during a stoppage in play. The soccer player who has been substituted takes no further part in a match (Rey et al., 2015). Substitutions are used for several reasons during a match: to replace a player who has become injured, or punished with a yellow card and thus at risk of being dismissed (Ascari and Gagnepein, 2006), when a certain player is showing signs of fatigue (Reilly et al., 2008) or for tactical reasons (Hirotsu and Wright, 2002).
According to studies assessing changes in match running performance in professional soccer players, the reduction in players' performance caused by fatigue produces a decline of approximately $10 \%$ in the total distance covered between the first and the second half of a match (Bradley and Noakes, 2013; Mohr et al., 2003). In addition, the ability to perform high intensity efforts, which is an important indicator of physical performance, also showed reductions of between $15-45 \%$ in the last $15 \mathrm{~min}$ of a match in comparison with the first four 15 min periods in professional soccer players (Bradley and Noakes, 2013; Mohr et al., 2003; Rampini et al., 2007). Reilly et al. (2008) proposed the use of substitute players as an alternative strategy to counteract fatigue. Several studies suggest that players introduced into a soccer match as a substitute

1 - Faculty of Education and Sports Sciences, University of Vigo, Campus Universitario A Xunqueira s/n, Pontevedra, Spain.

2 - Faculty of Education, University of Vigo, Campus Universitario As Lagoas s/n, Ourense, Spain. 
would cover equivalent or greater distance in high-intensity running (HIR) than the replaced players or other team members in the same time period (Bradley et al., 2014; Carling et al., 2010). Mohr et al. (2003) showed that the work-rate of substitutes who had been brought on before the 75th min of play was superior to that of players lasting the whole game for the final $15 \mathrm{~min}$. Furthermore, in the English Premier League Bradley et al. (2014) observed that substitute players covered greater HIR distance than substituted players and those who completed the entire match regardless of their position on the field, this was particularly evident in attackers. However, Bradley et al. (2014) pointed out the need to increase the scientific evidence on the role of the substitute player, in order to understand their impact on the match at the technical, tactical and conditional level.

The variation of the conditional performance in professional soccer players during a competitive season has been widely studied in the scientific literature (Malone et al., 2015; Mohr and Krustrup, 2014; Rampinini et al., 2007). Rampinini et al. (2007) analysed variations in match running performance throughout an entire competitive season in Italian Serie A professional soccer players. For match performance variables such as total distance, high intensity running and very high intensity running, it was observed that as the end of the season neared, players covered greater distances for each of these variables in comparison to the beginning of the season. In a study of 30 professional players from the English Premier League, Malone et al. (2015) analysed the training load during a season, including the preseason and in-season phases. In this study a reverse effect was presented when compared to the results obtained by Rampinini et al. (2007), as observed in the final third of the season when the training loads were reduced, leading to a reduction of the total distance covered by players for this period. Another study on semiprofessional soccer players showed that physical fitness of players changed as the season progressed (Mohr and Krustrup, 2014), showing a greater distance covered in the mid-season compared to the start and the end of the season in the Yo-Yo IR1 and Yo-Yo IR2 tests. Although there is scientific literature that shows the variation of physical performance for different variables at different periods of the season in soccer players, no research has analysed how running performance of substitute players is modified.

Currently, to our knowledge no research has analyzed performance of substitute players participating in La Liga or variation in their running match performance depending on the time of the season. Cultural differences and different training regimes in each league cause the presence of a profile of dissimilar physical and technical performance for each competition (Dellal et al., 2011); this is why the need emerges to compare the physical performance of substitute players with the substituted players and the ones who have completed the whole match. Hence the aims of this study were to (a) evaluate match running performances of substitute players versus those replaced or completing the entire match, (b) determine match running performances of substitute players across different playing positions and (c) examine variations in matchrelated running performance in substitute players throughout the entire competitive season.

\section{Methods}

\section{Participants}

The sample was composed of 943 observations of professional male soccer players. Participants played in the first division of the Spanish League (La Liga) during the 2014-2015 season. To ensure players and team's confidentiality, all data were anonymised. We, the authors, have no conflicts of interest that are directly relevant to the content of this article.

\section{Measures}

Match performance variables were classified in different categories and speed thresholds: low intensity $\left(0-14 \mathrm{~km} \cdot \mathrm{h}^{-1}\right)$, medium intensity (14-21 $\left.\mathrm{km} \cdot \mathrm{h}^{-1}\right)$, high intensity running (> $\left.21 \mathrm{~km} \cdot \mathrm{h}^{-1}\right)$, very high intensity running (21-24 $\mathrm{km} \cdot \mathrm{h}^{-1}$ ) and sprinting $\left(>24 \mathrm{~km} \cdot \mathrm{h}^{-1}\right)$. Absolute distances $(\mathrm{m})$ were converted to relative distances covered per unit of time $\left(\mathrm{m} \cdot \mathrm{min}^{-1}\right)$. Total distances represented the summation of distance in different categories. The peak speed reached during the match was defined as maximal running speed.

Procedures

The 2014-2015 season matches were analysed using a multiple-camera computerized 
tracking system Mediacoach (Mediapro \& LFP, Madrid, Spain). This semi-automatic system uses Tracab $^{\circledR}$ and OPTA $^{\circledR}$ technologies to capture player's activities during matches at 25 frames per second by 16 cameras positioned at the roof level and analysed using proprietary software. The reliability and validity of similar videotracking systems had been demonstrated previously (Di Salvo et al., 2006). Also the reliability of OPTA ${ }^{\circledR}$ Sportsdata had been assessed before (Liu et al., 2013). The players were divided into three different groups (Bradley et al., 2014): players who completed the entire match $(n=519)$, players who were replaced $(\mathrm{n}=212)$ and substitute players $(n=212)$. According to Carling et al. (2010) players were classified based on their skills as central defenders, fullbacks, central midfielders, wide midfielders and attackers. The seasonal variations were investigated comparing the mean of match performance variables during three periods of the competitive season (Rampinini et al., 2007): start (13 matches from August to November), mid (12 matches from December to February) and end (13 matches from March to May).

\section{Statistical analysis}

All analyses were conducted using the statistical package SPSS for Macintosh (version 21.0, Chicago, IL, USA). Data were provided as mean \pm standard deviation. Data normality was assessed using the Kolmogorov-Smirnov test. Analysis of variance with one-way ANOVA and post hoc Bonferroni tests were used to explore differences in running match performance of professional soccer players who played the entire match versus replaced and substitute players. Moreover, independent samples t-test was used to examine the interaction between measures of performance across playing positions. According to Cohen (1988), effect sizes were classified as small $(0.00 \leq \mathrm{d} \leq 0.49)$, medium $(0.50 \leq \mathrm{d} \leq 0.79)$, and large $(\mathrm{d} \geq 0.80)$. If the $90 \% \mathrm{CI}$ overlapped positive and negative values, the magnitude was deemed unclear; otherwise the magnitude was deemed to be observed magnitude. Thresholds used for assigning quantitative terms to chances were as follows: $<1 \%$ almost certainly not, $<5 \%$ very unlikely, $<25 \%$ unlikely, $<50 \%$ possibly not, $>50 \%$ possibly, $>75 \%$ likely, $>95 \%$ very likely, and $>99 \%$ almost certain (Batterham and Hopkins, 2006). For each of the tests used in this research the significance value was set at the $p<0.05$ level.

\section{Results}

A total of 212 substitutions were analysed, involving 9 central defenders, 15 fullbacks, 56 central midfielder, 77 wide midfielders and 55 attackers. Substitutions were grouped into $5 \mathrm{~min}$ periods (Figure 1). The number of substitutions made during the first and the second half did not present a homogeneous distribution, increasing the number of substitutions made during the second half $(p<0.001)$. In Figure 1, we can see how most of the substitutions were made at rest (at halftime) and during the time period from the $55^{\text {th }}$ to $85^{\text {th }} \mathrm{min}$ of the second half. The profile of the substitutions does not present a homogeneous distribution, showing a higher $(p<0.001)$ number of offensive (attackers, central and wide midfielders) than defensive substitutions (central defenders and fullbacks).

Table 1 displays match-performance characteristics of players using an independentmeasures analysis. The substitutes and replaced players covered greater $(p<0.01)$ total distance (ES: 0.44 to 0.44 , respectively), at medium intensity (ES: 0.72 to 0.37 , respectively), high speed (ES: 0.71 to 0.45 , respectively), and while sprinting (ES: 0.48 to 0.45 , respectively) and HIR (ES: from 0.67 to 0.51 , respectively) than players who completed the entire match. The substitute players covered greater distance $(p<0.01)$ at medium intensity ( $E S=0.38$ ) and higher speed (ES $=0.30$ ) than replaced players. Maximal running speed was higher $(p<0.01)$ in players completing the entire match and those replaced compared with substitutes. There was a trend for an improvement in running performance independent of the position in substitute players in comparison to the starter players represented by almost certain small to almost certain moderate improvements (ES range: 0.44-0.72) except for low intensity and maximal running speed.

Furthermore, in Table 1 the results of running performance depending on the field position are presented. In central midfielders, almost certain small differences were observed for high speed and HIR in substitutes versus starter players (ES: 0.54). A general trend for an increased distance covered at high speed and HIR was observed in central midfielders' substitutes. In attackers, a likely small difference was observed in 
HIR between substitutes and players who started the match (ES: 0.45). However, no differences were found between substitutes of central defenders, fullbacks and wide midfielders compared with players who completed the entire match or that were replaced. The maximal running speeds attained during the entire match were $(p<0.01)$ greater in all playing positions compared with substitutes.

Table 2 compares match-running performance by substitutes, substituted players and those who completed the whole game for each of the periods of the competitive season. Substitute players covered greater $(p<0.01)$ distance at medium intensity (ES: 0.65), high speed (ES: 0.75), while sprinting (ES: 0.45) and HIR (ES: 0.68) than players who completed the entire match at the beginning of the competitive season. At the mid-season, substitute players covered greater $(p<0.01)$ total distance (ES: 0.52), distance at medium intensity (ES: 0.83), high speed (ES: 0.77), while sprinting (ES: 0.71) and HIR (ES: 0.88) than players who completed the entire match. In addition, an increase was observed during the mid-season in running performance of substitute players for the variables of total distance, high speed and HIR compared with the start of the competitive season. At the end of the competitive period, substitute players covered greater $(p<0.01)$ total distance (ES: 0.64), distance at medium intensity (ES: 0.73), high speed (ES: 0.58) and HIR (ES: 0.44) than players who completed the entire match. At the end of the season, a decrease in distance covered for all variables of running performance was observed, except for the total distance and low intensity compared with the start and the end of the season.

\section{Discussion}

To our knowledge, there are only two previous studies that analysed physical performance of substitute players compared to replaced players and those who played the entire match in the English Premier League and French Ligue 1 (Bradley et al., 2014; Carling et al., 2010). Therefore, and due to the differences in the physical and technical indicators found among domestic competitions belonging to the European continent by Dellal et al. (2011), this study aimed to provide evidence about physical performance of substitute players compared to those players who were replaced or played the entire match in La Liga.

The results obtained in this study showed that most substitutions during matches played in La Liga were performed at half-time and between the $55^{\text {th }}$ and $85^{\text {th }}$ min of the second half. In addition, it was observed that coaches commonly performed offensive substitutions, involving attackers, central midfielders and wide midfielders. These findings are in agreement with those obtained by Del Corral et al. (2007), during the 2004-2005 season in La Liga. Likewise, the results were similar to those obtained in other international and national competitions such as the UEFA Champions League and English Premier League in which a powerful tendency to increase the number of substitutions made by a coach at half-time and during the second half was revealed, with offensive players being the ones most frequently replaced (Bradley et al., 2014; Rey et al., 2015). Hence, the timing of replacement as well as the substitution type seems to have a predetermined pattern, manifesting itself similarly regardless of the competition.

The high-intensity running variable is considered an important indicator of physical performance in professional soccer (Carling, 2010; Di Salvo et al., 2009; Krustrup et al., 2003). Research demonstrated that the number of performed sprints, HIR and distance covered are lower in the second half than in the first half of the game (Bangsbo, 1994; Bradley et al., 2010; Morhr et al., 2003). The literature proposes strategic use of substitute players as a means to reduce team fatigue (Reilly et al., 2008). Thus, substitute players covered $25 \%$ more ground while running at high intensity during the final 15 min than the other players (Mohr et al., 2003). In a previous study developed with players belonging to the English Premier League, it was observed that players introduced as substitutes were able to cover by $10 \%$ and $27 \%$ more distance in HIR than players who were replaced or played the whole game (Bradley et al., 2014). Similar findings were also reported in other team sports like rugby (Lacome et al., 2016), indicating that the pacing strategies adopted by team sports players to manage energy resources and anticipate fatigue may differ according to the starting status. 
Table 1

Independent-measures analysis of match performance of substitute players versus those who completed the entire match or were replaced.

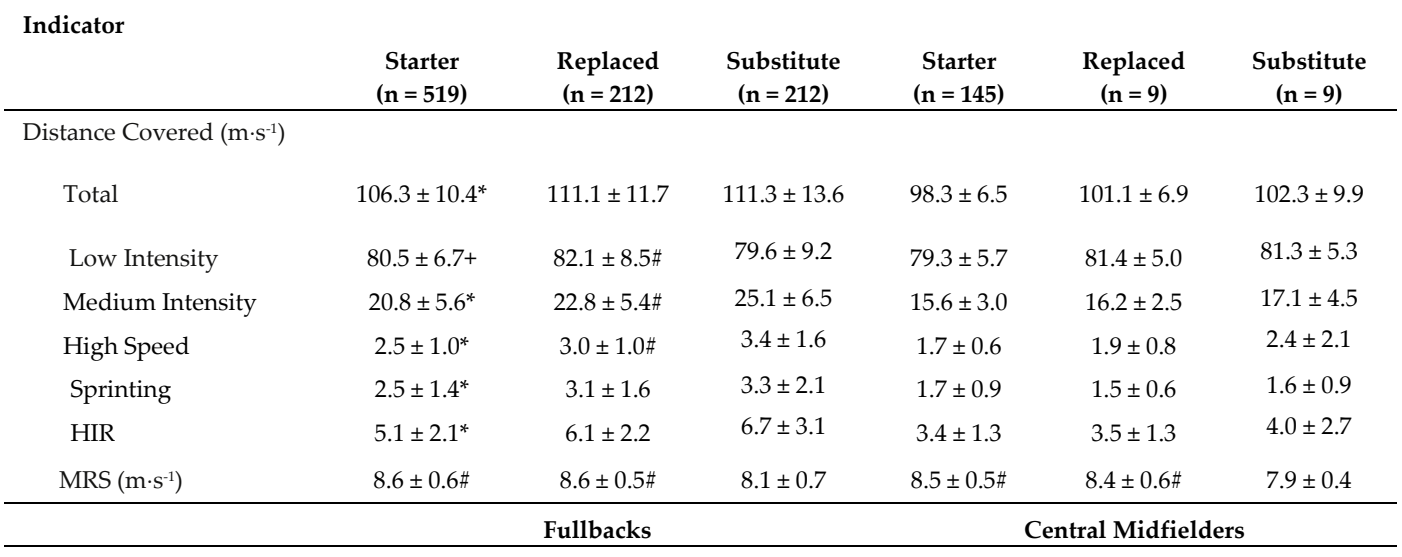

\begin{tabular}{|c|c|c|c|c|c|c|}
\hline & $\begin{array}{c}\text { Starter } \\
(\mathrm{n}=129)\end{array}$ & $\begin{array}{c}\text { Replaced } \\
(\mathrm{n}=18)\end{array}$ & $\begin{array}{c}\text { Substitute } \\
(\mathrm{n}=18)\end{array}$ & $\begin{array}{c}\text { Starter } \\
(\mathrm{n}=134)\end{array}$ & $\begin{array}{c}\text { Replaced } \\
(\mathrm{n}=56)\end{array}$ & $\begin{array}{c}\text { Substitute } \\
(\mathrm{n}=56)\end{array}$ \\
\hline \multicolumn{7}{|l|}{ Distance Covered $\left(\mathrm{m} \cdot \mathrm{s}^{-1}\right)$} \\
\hline Total & $105.7 \pm 7.0$ & $107.6 \pm 7.1$ & $103.9 \pm 16.5$ & $114.2 \pm 8.1$ & $115.3 \pm 8.8$ & $113.5 \pm 13.7$ \\
\hline Low Intensity & $78.8 \pm 5.1$ & $80.3 \pm 4.2$ & $76.8 \pm 10.6$ & $83.9 \pm 5.8 \#$ & $84.9 \pm 5.9 \#$ & $80.6 \pm 9.4$ \\
\hline Medium Intensity & $20.5 \pm 3.4$ & $20.5 \pm 4.6$ & $20.6 \pm 6.7$ & $25.9 \pm 4.9$ & $26.0 \pm 5.3$ & $27.5 \pm 5.9$ \\
\hline High Speed & $2.9 \pm 0.9$ & $3.1 \pm 0.9$ & $3.2 \pm 1.6$ & $2.5 \pm 0.9 \#$ & $2.5 \pm 0.9 \#$ & $3.2 \pm 1.8$ \\
\hline Sprinting & $3.5 \pm 1.3$ & $3.6 \pm 1.1$ & $3.3 \pm 2.7$ & $1.8 \pm 1.0$ & $1.9 \pm 1.1$ & $2.2 \pm 1.6$ \\
\hline HIR & $6.4 \pm 1.8$ & $6.8 \pm 1.6$ & $6.6 \pm 4.0$ & $4.3 \pm 1.6 \#$ & $4.4 \pm 1.7 \ddagger$ & $5.4 \pm 2.8$ \\
\hline \multirow[t]{2}{*}{$\operatorname{MRS}\left(\mathrm{m} \cdot \mathrm{s}^{-1}\right)$} & $8.9 \pm 0.4 \#$ & $8.8 \pm 0.5$ & $8.1 \pm 0.9$ & $8.3 \pm 0.5 \#$ & $8.2 \pm 0.5 \#$ & $7.9 \pm 0.6$ \\
\hline & \multicolumn{4}{|c|}{ Wide Midfielders } & Attackers & \\
\hline
\end{tabular}

\begin{tabular}{lcccccc} 
& $\begin{array}{c}\text { Starter } \\
(\mathbf{n}=\mathbf{6 3})\end{array}$ & $\begin{array}{c}\text { Replaced } \\
(\mathbf{n}=\mathbf{8 2})\end{array}$ & $\begin{array}{c}\text { Substitute } \\
(\mathbf{n}=\mathbf{8 2})\end{array}$ & $\begin{array}{c}\text { Starter } \\
(\mathbf{n}=\mathbf{4 8})\end{array}$ & $\begin{array}{c}\text { Replaced } \\
(\mathbf{n}=\mathbf{5 5})\end{array}$ & $\begin{array}{c}\text { Substitute } \\
(\mathbf{n}=\mathbf{5 5})\end{array}$ \\
\hline Distance Covered $\left(\mathrm{m} \cdot \mathrm{s}^{-1}\right)$ & & & & & & \\
$\quad$ & $110.6 \pm 10.7$ & $112.1 \pm 13.3$ & $113.4 \pm 10.7$ & $105.1 \pm 13.8$ & $107.9 \pm 12.0$ & $110.2 \pm 15.2$ \\
Total & $80.6 \pm 7.6$ & $82.6 \pm 9.6$ & $79.8 \pm 7.8$ & $79.0 \pm 10.5$ & $79.1 \pm 9.7$ & $78.7 \pm 10.7$ \\
Low Intensity & $23.3 \pm 5.0 \#$ & $22.9 \pm 5.3 \#$ & $26.2 \pm 5.0$ & $19.6 \pm 4.3 \#$ & $21.2 \pm 4.2 \ddagger$ & $23.9 \pm 7.0$ \\
Medium Intensity & $3.2 \pm 0.8$ & $3.2 \pm 1.0$ & $3.5 \pm 1.5$ & $3.1 \pm 0.9$ & $3.3 \pm 0.9$ & $3.7 \pm 1.5$ \\
High Speed & $3.4 \pm 1.3$ & $3.5 \pm 1.6$ & $3.9 \pm 2.2$ & $3.4 \pm 1.4$ & $4.2 \pm 1.5$ & $3.9 \pm 2.0$ \\
Sprinting & $6.6 \pm 1.8$ & $6.6 \pm 2.1$ & $7.4 \pm 2.9$ & $6.5 \pm 2.0 \pm$ & $7.5 \pm 2.0$ & $7.7 \pm 3.0$ \\
HIR & $8.7 \pm 0.5 \#$ & $8.7 \pm 0.5 \#$ & $8.3 \pm 0.7$ & $8.8 \pm 0.4 \#$ & $8.8 \pm 0.4 \#$ & $8.3 \pm 0.7$ \\
MRS $\left(\mathrm{m} \cdot \mathrm{s}^{-1}\right)$ & &
\end{tabular}

Abbreviations: HIR, high intensity running; $M R S$, maximal running speed.

${ }^{*}$ Different from replaced and substitute players, $p<.01$. +Different from replaced players, $p<.05$. $\ddagger$ Different from substitute players, $p<.05$. \#Different from substitute players, $p<.01$ 
Table 2

Seasonal variations of match performance of substitute players versus those who completed the entire match or were replaced.

\begin{tabular}{|c|c|c|c|}
\hline & \multicolumn{3}{|c|}{ Early-Season } \\
\hline Indicator & $\begin{array}{c}\text { Starter } \\
(\mathrm{n}=179)\end{array}$ & $\begin{array}{l}\text { Replaced } \\
(\mathrm{n}=75)\end{array}$ & $\begin{array}{l}\text { Substitute } \\
(\mathrm{n}=75)\end{array}$ \\
\hline \multicolumn{4}{|c|}{ Distance Covered $\left(\mathrm{m} \cdot \mathrm{s}^{-1}\right)$} \\
\hline Total & $108.0 \pm 10.5+$ & $112.3 \pm 12.7$ & $110.9 \pm 17.1$ \\
\hline Low Intensity & $81.8 \pm 7.0$ & $82.4 \pm 9.2$ & $79.2 \pm 12.1$ \\
\hline Medium Intensity & $21.2 \pm 5.6^{*}$ & $23.9 \pm 5.2$ & $25.0 \pm 6.5$ \\
\hline High Speed & $2.5 \pm 1.0+\#$ & $2.9 \pm 1.0 \ddagger$ & $3.5 \pm 1.8$ \\
\hline Sprinting & $2.4 \pm 1.4+\#$ & $3.1 \pm 1.6$ & $3.2 \pm 2.0$ \\
\hline HIR & $4.9 \pm 2.2^{*}$ & $6.0 \pm 2.2$ & $6.7 \pm 3.3$ \\
\hline \multirow[t]{3}{*}{$\operatorname{MRS}\left(\mathrm{m} \cdot \mathrm{s}^{-1}\right)$} & $8.6 \pm 0.5 \#$ & $8.6 \pm 0.5 \#$ & $8.2 \pm 0.8$ \\
\hline & \multicolumn{3}{|c|}{ Middle-Season } \\
\hline & $\begin{array}{c}\text { Starter } \\
(\mathrm{n}=163)\end{array}$ & $\begin{array}{c}\text { Replaced } \\
(n=65)\end{array}$ & $\begin{array}{l}\text { Substitute } \\
(\mathrm{n}=65)\end{array}$ \\
\hline
\end{tabular}

Distance Covered $\left(\mathrm{m} \cdot \mathrm{s}^{-1}\right)$

\begin{tabular}{|c|c|c|c|}
\hline Total & $105.6 \pm 11.2^{*}$ & $110.9 \pm 12.2$ & $111.4 \pm 11.1$ \\
\hline Low Intensity & $79.3 \pm 7.1$ & $81.6 \pm 9.4$ & $78.5 \pm 7.6$ \\
\hline Medium Intensity & $21.2 \pm 5.7 \#$ & $22.4 \pm 4.8 \#$ & $25.8 \pm 5.4$ \\
\hline High Speed & $2.6 \pm 1.0^{*}$ & $3.3 \pm 0.9$ & $3.5 \pm 1.5$ \\
\hline Sprinting & $2.5 \pm 1.3^{*}$ & $3.6 \pm 1,7$ & $3.6 \pm 2.0$ \\
\hline HIR & $5.1 \pm 2.0^{*}$ & $6.9 \pm 2.3$ & $7.1 \pm 2.8$ \\
\hline \multirow[t]{3}{*}{$\operatorname{MRS}\left(\mathrm{m} \cdot \mathrm{s}^{-1}\right)$} & $8.5 \pm 0.5 \#$ & $8.7 \pm 0.5 \#$ & $8.2 \pm 0.5$ \\
\hline & \multicolumn{3}{|c|}{ End-Season } \\
\hline & $\begin{array}{c}\begin{array}{c}\text { Starter } \\
(\mathrm{n}=177)\end{array} \\
\end{array}$ & $\begin{array}{c}\text { Replaced } \\
(\mathrm{n}=72)\end{array}$ & $\begin{array}{c}\text { Substitute } \\
(\mathrm{n}=72)\end{array}$ \\
\hline \multicolumn{4}{|c|}{ Distance Covered $\left(\mathrm{m} \cdot \mathrm{s}^{-1}\right)$} \\
\hline Total & $105.4 \pm 8.9^{*}$ & $110.1 \pm 9.7$ & $111.7 \pm 11.9$ \\
\hline Low Intensity & $80.4 \pm 5.4$ & $82.6 \pm 6.2$ & $81.1 \pm 6.9$ \\
\hline Medium Intensity & $19.8 \pm 5.5 \#$ & $22.0 \pm 6.1$ & $24.3 \pm 7.4$ \\
\hline High Speed & $2.5 \pm 0.9 \#$ & $2.7 \pm 1.0$ & $3.2 \pm 1.6$ \\
\hline Sprinting & $2.6 \pm 1.5$ & $2.9 \pm 1.5$ & $3.1 \pm 2.4$ \\
\hline HIR & $5.1 \pm 2.2 \#$ & $5.5 \pm 2.2$ & $6.3 \pm 3.4$ \\
\hline $\operatorname{MRS}\left(\mathrm{m} \cdot \mathrm{s}^{-1}\right)$ & $8.6 \pm 0.5$ & $8.5 \pm 0.5$ & $8.0 \pm 0.8$ \\
\hline
\end{tabular}

Abbreviations: HIR, high intensity running; MRS, maximal running speed.

${ }^{*}$ Different from replaced and substitute players, $p<.01$.

+ Different from replaced players, $p<.05$.

$\ddagger$ Different from substitute players, $p<.05$. \#Different from substitute players, $p<.01$ 


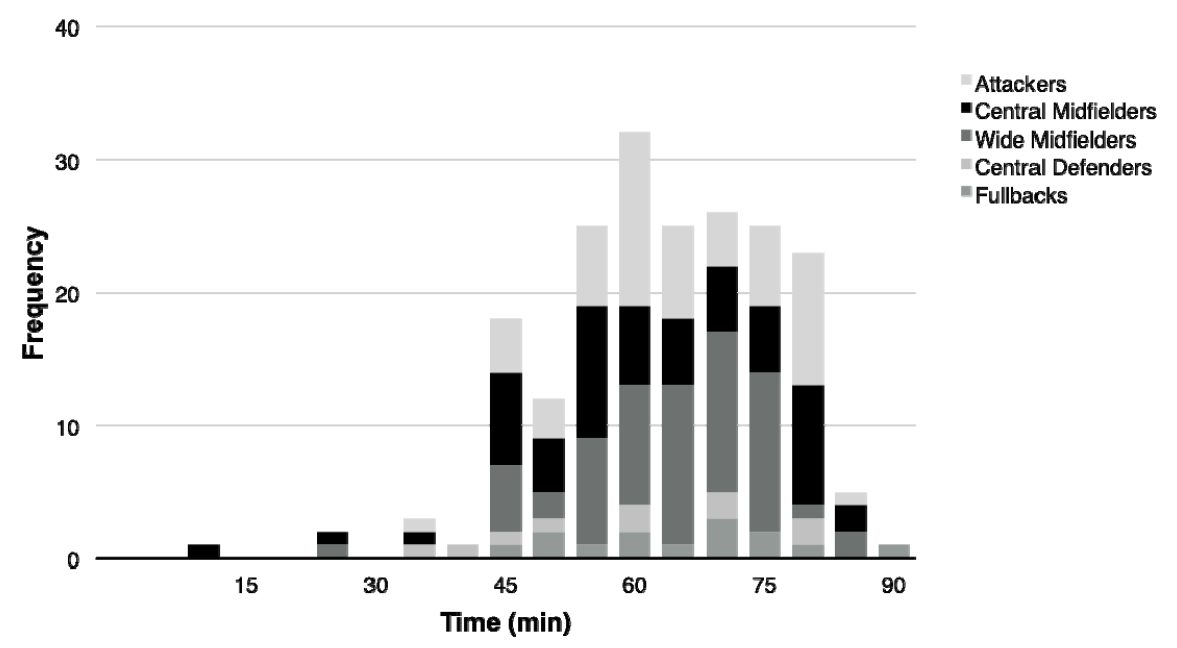

Figure 1

Histogram of all substitutions

These findings are in agreement with the current study, as players entering the match as substitutes covered by $9 \%$ and $24 \%$ more distance while HIR than those replaced and completing the entire match, respectively. Waldron and Highton (2014) in a review of the pacing strategies in highintensity team sports indicate that soccer players who start the match present a pacing scheme which is referred to as "slow positive", this strategy means that the intensity of running will progressively decline across the match. However, some substitute players use a pacing strategy referred to as "one bout, all out" (Waldron and Highton, 2014), this strategy allows them to maintain HIR for a short period of time, thereby obtaining a positive correlation between HIR and the proximity of the substitution to the end of the match (Bradley and Noakes, 2013).

According to the position of the player variable, the results of this research revealed how substitute players who performed in the position of a central midfielder and attacker were able to significantly increase the distance covered in HIR compared to players who completed the entire match or who were replaced. Furthermore,
Carling et al. (2010) demonstrated that midfield substitutes covered greater overall distances and high-intensity distance, and had a lower recovery time between high-intensity bouts when compared with other midfield teammates who continued the match. However, substitute attackers were not able to increase the distance covered at high-intensity compared to players who completed the entire match, also, they covered a lower distance in the first ten minutes as a substitute compared to their usual work-rate profile in the opening ten minutes when starting matches. In players from the English Premier League, Bradley et al. (2014) observed an increase in the distance covered in HIR for the position of a central defender, central midfielder, wide midfielder and attacker, yet this finding was not observed in fullbacks. Therefore, an uneven conditional performance between substitute players from European leagues is observed, which could be explained, as Dellal et al. (2011) suggest, by cultural differences between professional leagues and playing positions.

Currently, there are consistent findings that physical fitness in professional soccer players 
seems to improve significantly during the preseason period (Krustrup et al., 2006). To our knowledge, no study has analysed performance variations of substitute players at different periods of the season. At the start of the season, it was observed that substitute players obtained greater performance in running at medium intensity, high speed, sprinting and HIR in comparison to players who completed the entire match, but it should be noted that they only increased their performance when compared to the players substituted in the variable of high speed. The results seem to indicate that substitute players reach their best running performance during the mid-season, being able to run a greater distance relative to the overall variables: distance, medium intensity running, high speed running, sprinting and HIR than players which complete the entire match. When comparing performance of substitutes with replaced players, only significant differences for the variables of medium intensity running and maximal running speed were found. However, at the end of the season the relative distances covered were lower than at the start and the mid-season, in substitute players, the ones who were substituted and those who completed the entire match. For this period of the season no significant differences were observed in match running performance between substituted players and those who completed the whole game, except for the total distance variable. While, during the end of the season, substitute players covered greater relative distance than players who completed the entire match for the following variables: total distance, medium intensity running, high speed running and HIR. These findings are in agreement with Mohr and Krustrup (2014) who analysed the evolution of aerobic and anaerobic performance of semiprofessional players for different periods of the season. These authors observed that during the mid-season players covered greater distances and had lower values of the maximum heart rate during the start of the season and the mid-season. During the mid-season, soccer players improved performance in activities involving stretchshortening cycles, such as sprints, jumps and actions that involved some degree of agility (Silva et al., 2011). The decrease in performance at the end of the season could be due to decreased training loads (Malone et al., 2015) and lower aerobic power values seen during this period (Mohr and Krustrup, 2014). Reducing training loads could have a negative effect on performance of substitute players who play regularly since they have fewer minutes of play than substituted players and players who complete the entire match. Consequently, one would expect that players who play the entire match or are replaced reduce their physical performance during matches because of residual fatigue accumulated throughout the season, however, players who play as substitutes may suffer, since they compete for a limited number of minutes and their training loads are also reduced.

Most of the substitutions were performed during the second half of a match and between the 55th and 85th min in the Spanish league, usually presenting an offensive character, thus involving players who develop the specific position of attackers, central and wide midfielders. Substitute players increased the work-rate compared to players who played the entire match and those who were replaced. Position-specific trends indicated that attackers and central midfielders increased the distance covered at high-intensity running compared to their peers who played the entire match. During the competitive season, it was observed that substitute players attained greater match running performance during the mid-season period, allowing them to cover more distance for the different variables of running performance compared to the start of the season and the end of the season.

\section{Acknowledgements}

This study was supported by grants Xunta de Galicia.

\section{References}

Ascari G, Gagnepain P. Spanish football. J Sports Econom, 2006; 7: 76-89

Bangsbo J. The physiology of soccer with special reference to intense intermittent exercise. Acta Physiol Scand 
Suppl, 1994; 619: 1-155

Batterham A, Hopkins WG. Making meaningful inferences about magnitudes. Int J Sports Physiol Perform, 2006; 1 : 50-57

Bradley PS, Noakes TD. Match running performance fluctuations in elite soccer: Indicative of fatigue, pacing or situational influences? J Sports Sci, 2013; 31: 1627-1638

Bradley PS, Di Mascio M, Peart D, Olsen P, Sheldon B. High-intensity activity profiles of elite soccer players at different performance levels. J Strength Cond Res, 2010; 24: 2343-2351

Bradley PS, Lago-Peñas C, Rey E. Evaluation of the match performances of substitution players in elite soccer. Int J Sports Physiol Perform, 2014; 9: 415-424

Carling C. Analysis of physical activity profiles when running with the ball in a professional soccer team. J Sports Sci, 2010; 28: 319-326

Carling C, Espié V, Le Gall F, Bloomfield J, Jullien H. Work-rate of substitutes in elite soccer: A preliminary study. J Sci Med Sport, 2010; 13: 253-255

Cohen J. Statistical power analysis for the behavioural sciences (2nd ed). Hillsdale, NJ: Lawrence Erlbaum; 1988

Del Corral J, Barros CP, Prieto-Rodriguez J. The determinants of soccer player substitutions: a survival analysis of the Spanish soccer league. J Sports Econom, 2008; 9: 160-172

Dellal A, Chamari K, Wong DP, Ahmaidi S, Keller D, Barros R, Bisciotti GN, Carling, C. Comparison of physical and technical performance in European soccer match-play: FA Premier League and La Liga. Eur J Sport Sci, 2011; 11: 51-59

Di Salvo V, Collins A, McNeill B, Cardinale M. Validation of Prozone: A new video-based performance analysis system. Int J Perform Anal Sport, 2006; 6: 108-119

Di Salvo V, Gregson W, Atkinson G, Tordoff P, Drust B. Analysis of high intensity activity in Premier League soccer. Int J Sports Med, 2009; 30: 205-212

Fédération Internationale de Football Association. Laws of the Game: Law 3. Zurich: FIFA, 17-21; 2014

Hirotsu N, Wright M. Using a Markov process model of an association football match to determine the optimal timing of substitution and tactical decisions. J Oper Res Soc, 2002; 53: 88-96

Krustrup P, Mohr M, Amstrup T, Rysgaard T, Johansen J, Steensberg A, Pedersen PK, Bangsbo J. The yo-yo intermittent recovery test: physiological response, reliability, and validity. Med Sci Sports Exerc, 2003; 35: 697-705

Krustrup P, Mohr M, Nybo L, Jensen JM, Nielsen JJ, Bangsbo J. The Yo-Yo IR2 test: physiological response, reliability, and application to elite soccer. Med Sci Sports Exerc, 2006; 38: 1666-1673

Lacome M, Piscione J, Hager JP, Carling C. Analysis of running and technical performance in substitute players in international male Rugby Union competition. Int J Sports Physiol Perform, 2016; 11: 783-792

Liu H, Hopkins W, Gómez MA, Molinuevo JS. Inter-operator reliability of live football match statistics from OPTA Sports data. Int J Perform Anal Sport, 2013; 13: 803-821

Malone JJ, Di Michele R, Morgans R, Burgess D, Morton JP, Drust B. Seasonal training-load quantification in elite English premier league soccer players. Int J Sports Physiol Perform, 2015; 10: 489-497

Mohr M, Krustrup P. Yo-Yo intermittent recovery test performances within an entire football league during a full season. J Sports Sci, 2014; 32: 315-327

Mohr M, Krustrup P, Bangsbo J. Match performance of high-standard soccer players with special reference to development of fatigue. J Sports Sci, 2003: 21; 519-528

Rampinini E, Coutts AJ, Castagna C, Sassi R, Impellizzeri FM. Variation in top level soccer match 
performance. Int J Sports Med, 2007; 28: 1018-1024

Reilly T, Drust B, Clarke N. Muscle fatigue during football match-play. Sports Med, 2008: 38; 357-367

Rey E, Lago-Ballesteros J, Padrón-Cabo A. Timing and tactical analysis of player substitutions in the UEFA Champions League. Int J Perform Anal Sport, 2015; 15: 840-850

Silva JR, Magalhães JF, Ascensão AA, Oliveira EM, Seabra AF, Rebelo AN. Individual match playing time during the season affects fitness-related parameters of male professional soccer players. J Strength Cond Res, 2011; 25: 2729-2739

Waldron M, Highton J. Fatigue and pacing in high-intensity intermittent team sport: an update. Sports Med, 2014; 44; 1645-1658

\section{Corresponding author:}

\section{Alexis Padrón-Cabo}

Faculty of Education and Sports Sciences

University of Vigo

Campus Universitario A Xunqueira s/n, Pontevedra, 36005, Spain

Tel: +34 670088148;

Fax: +34 986801701;

E-mail: alexiscabo03@gmail.com 